\title{
Vitamin D to prevent exacerbations of COPD: systematic review and meta-analysis of individual participant data from randomised controlled trials
}

\author{
David A Jolliffe, ${ }_{1}^{1}$ Lauren Greenberg, ${ }^{1}$ Richard L Hooper, ${ }^{1}$ Carolien Mathyssen, ${ }^{2}$ \\ Rachida Rafiq, ${ }^{3}$ Renate T de Jongh, ${ }^{3}$ Carlos A Camargo, ${ }^{4}$ Christopher J Griffiths, ${ }^{1,5}$ \\ Wim Janssens, ${ }^{2}$ Adrian R Martineau ${ }^{1,5}$
}

\begin{abstract}
- Additional material is published online only. To view please visit the journal online (http://dx.doi.org/10.1136/ thoraxjnl-2018-212092).

For numbered affiliations see end of article.
\end{abstract}

\section{Correspondence to} Professor Adrian R Martineau, The Centre for Primary Care and Public Health, Blizard Institute, Barts and The London School of Medicine and Dentistry, Queen Mary University of London, London E1 2AB, UK ; a.martineau@qmul.ac.uk

WJ and ARM contributed equally.

Received 19 May 2018 Revised 20 November 2018 Accepted 26 November 2018 Published Online First 10 January 2019

\begin{abstract}
Background Randomised controlled trials (RCTs) of vitamin D to prevent COPD exacerbations have yielded conflicting results.

Individual participant data meta-analysis could identify factors that explain this variation.

Methods PubMed, Embase, the Cochrane Central Register of Controlled Trials and Web of Science were searched from inception up to and including 5 October 2017 to identify RCTs of vitamin D supplementation in patients with COPD that reported incidence of acute exacerbations. Individual participant data meta-analysis was performed using fixed effects models adjusting for age, sex, Global Initiative for Chronic Obstructive Lung Disease spirometric grade and trial.
\end{abstract}

Results Four eligible RCTs (total 560 participants) were identified; individual participant data were obtained for 469/472 (99.4\%) participants in three RCTs. Supplementation did not influence overall rate of moderate/severe COPD exacerbations (adjusted incidence rate ratio (alRR) $0.94,95 \% \mathrm{Cl} 0.78$ to 1.13). Prespecified subgroup analysis revealed that protective effects were seen in participants with baseline 25-hydroxyvitamin D levels $<25 \mathrm{nmol} / \mathrm{L}$ (alRR 0.55, $95 \% \mathrm{Cl} 0.36$ to 0.84 ) but not in those with baseline 25-hydroxyvitamin D levels $\geq 25 \mathrm{nmol} / \mathrm{L}$ (aIRR 1.04, 95\% $\mathrm{Cl} 0.85$ to 1.27 ; p for interaction=0.015). Vitamin D did not influence the proportion of participants experiencing at least one serious adverse event (adjusted OR 1.16, $95 \% \mathrm{Cl} 0.76$ to 1.75 ).

Conclusions Vitamin D supplementation safely and substantially reduced the rate of moderate/ severe COPD exacerbations in patients with baseline 25 -hydroxyvitamin $D$ levels $<25 \mathrm{nmol} / \mathrm{L}$ but not in those with higher levels.

Trial registration number CRD42014013953.

\section{INTRODUCTION}

COPD affects more than 170 million people worldwide and caused an estimated 3.2 million deaths in $2015 .^{1}$ COPD mortality arises primarily during episodes of acute worsening of symptoms termed exacerbations: these may be classified as severe (ie, requiring emergency department attendance or hospitalisation) or moderate (ie, requiring treatment with systemic corticosteroids or antibiotics, or both, but not precipitating emergency department attendance or hospitalisation). ${ }^{2}$ COPD exacerbations

\section{Key messages}

What is the key question?

- Does vitamin D supplementation protect against moderate/severe COPD exacerbations in particular subgroups of patients?

What is the bottom line?

- Vitamin D supplementation reduces the rate of moderate/severe COPD exacerbations in patients with baseline 25 -hydroxyvitamin $D$ levels $<25 \mathrm{nmol} / \mathrm{L}$ but not in those with higher levels.

Why read on?

- Our findings support a strategy of routinely testing vitamin D status in patients with COPD who experience exacerbations and offering supplementation to those with circulating $25(\mathrm{OH}) \mathrm{D}$ concentrations of less than $25 \mathrm{nmol} / \mathrm{L}$.

are commonly triggered by respiratory viruses and bacteria, which increase airway inflammation. ${ }^{3}$ Vitamin D metabolites support the induction of antiviral and antimicrobial effector mechanisms and attenuate inflammatory responses. ${ }^{4}$ Moreover, meta-analyses of individual participant data (IPD) from randomised controlled trials (RCTs) have shown that vitamin $\mathrm{D}$ supplementation reduces risk of acute respiratory infections and exacerbations of asthma. ${ }^{67}$ Taken together, these lines of evidence suggest a potential role for vitamin D supplementation in prevention of COPD exacerbations. ${ }^{8}$ Vitamin $\mathrm{D}$ deficiency is common in patients with COPD, but consistent associations between circulating concentrations of 25 -hydroxyvitamin D $(25(\mathrm{OH}) \mathrm{D}$, the major vitamin $\mathrm{D}$ metabolite) and risk of COPD exacerbation have not been demonstrated..$^{9-12}$ Four double-blind placebo-controlled RCTs have been published, investigating effects of vitamin D supplementation on the risk of COPD exacerbation: of these, three reported no effect overall ${ }^{13-15}$ and one reported a protective effect overall. ${ }^{16}$ These RCTs have not previously been meta-analysed. We therefore set out to meta-analyse IPD from these studies to determine whether vitamin $\mathrm{D}$ supplementation reduces COPD exacerbation rate overall and to explore whether the effects of this intervention 
vary according to potential effect modifiers, including baseline vitamin $\mathrm{D}$ status.

\section{METHODS}

\section{Protocol and registration}

The methods for this systematic review and one-step and two-step meta-analysis were described in an outline protocol that was registered with the PROSPERO International Prospective Register of Systematic Reviews (http://www.crd.york.ac.uk/prospero/display_record.php? ID $=$ CRD42014013953). The outline protocol includes populations of people at risk of acute respiratory infection, and people with asthma and COPD, and defines the primary objective of the meta-analysis, namely to determine whether there are differential effects of vitamin D supplementation on incidence of acute respiratory infection and acute exacerbations of asthma and COPD. This systematic review and meta-analysis of IPD focuses on people with COPD. Systematic reviews and meta-analyses of IPD in people with asthma and in those at risk of acute respiratory infection have been previously published. ${ }^{67}$ Research ethics committee approval was not required in Belgium, the UK or the Netherlands to do the current meta-analysis. Findings are reported according to the Preferred Reporting Items for Systematic Reviews and Meta-Analyses guidelines for IPD meta-analysis. ${ }^{17}$

\section{Study selection}

Studies were eligible for inclusion if they were double-blind, placebo-controlled RCTs (ie, those in which both participants and investigators were unaware of participant allocation during the trial) of supplementation with vitamin $\mathrm{D}_{3}$ or vitamin $\mathrm{D}_{2}$ in patients with COPD, approved by a research ethics committee and reporting data on incidence of COPD exacerbation. All of these criteria were assessed to establish eligibility. Trials in which vitamin $\mathrm{D}_{3}$ or vitamin $\mathrm{D}_{2}$ were administered in combination with other micronutrients were excluded.

\section{Data sources and searches}

Two investigators (DAJ and ARM) searched PubMed, Embase, the Cochrane Central Register of Controlled Trials (CENTRAL) and Web of Science using the electronic search strategies described in the online supplementary appendix. We regularly updated our searches from database inception up to and including 5 October 2017. No language restrictions were imposed. We supplemented these searches by searching review articles and reference lists of trial publications. Collaborators were asked if they knew of any additional RCTs. Three investigators (ARM, DAJ and WJ) determined which studies met the eligibility criteria.

\section{Definition of outcomes}

The primary outcome of the meta-analysis was the rate of COPD exacerbations requiring treatment with systemic corticosteroids or antibiotics, or both (ie, those classified as moderate or severe by the Global Initiative for Chronic Obstructive Lung Disease (GOLD) guidelines). ${ }^{2}$ We selected exacerbation rate as primary outcome on the basis that this captured information about all exacerbations that arose; moreover, analysis of event rate allows for inclusion of data from trials having different follow-up times. Secondary outcomes were the time from first dose of study medication to first moderate or severe exacerbation; the proportion of participants with at least one moderate or severe exacerbation; the proportion of COPD exacerbations resulting in emergency department attendance or hospitalisation, or both (ie, exacerbations classified as severe by GOLD guidelines) ${ }^{2}$; the proportion of participants experiencing at least one serious adverse event, episode of hypercalcaemia or episode of renal stones; mean per cent predicted $\mathrm{FEV}_{1}$ and FVC at final follow-up; mean BMI at final follow-up; and mortality (COPD related and all cause).

\section{Data synthesis and analysis}

Methods for data extraction, quality assurance and risk of bias assessments for individual studies are presented in the online supplementary appendix. Effects of the intervention on event rates, dichotomous outcomes and time to first event were expressed as incidence rate ratios (IRRs), ORs and HRs, respectively. DAJ, LG and RLH analysed the data using STATA IC, V.12. Our IPD meta-analysis approach followed published guidelines. ${ }^{18}$ Initially, all studies were reanalysed separately; the original authors were asked to confirm accuracy of this reanalysis where it had been done previously, and any discrepancies were resolved. We did both one-step and two-step IPD meta-analyses for the primary outcome of rate of moderate or severe COPD exacerbation, at the request of the funder. For secondary outcomes, we did one-step IPD meta-analysis only. In the one-step approach, IPD from all studies were modelled simultaneously with fixed effects, adjusting for age, sex, GOLD spirometric grade ${ }^{2}$ and trial to obtain the pooled intervention effect with a 95\% CI. We analysed event rates using negative binomial regression; survival data using parametric survival models; and continuous outcomes using linear regression, additionally adjusted for baseline value. Proportions were analysed using logistic regression, additionally adjusted for duration of participant follow-up; for the analysis of the proportion of participants experiencing at least one exacerbation, participants who were lost to follow-up before they experienced a moderate or severe exacerbation were classified as not having had such an exacerbation. In the two-step approach, event rates were first analysed for each separate study independently using negative binomial regression, with adjustment for age, sex and GOLD spirometric grade, ${ }^{2}$ to produce an estimate of the treatment effect for that study. We then calculated a weighted average of the individual treatment effect estimates using the Mantel-Haenszel method and summarised heterogeneity using the $\mathrm{I}^{2}$ statistic.

\section{Exploration of variation in effects}

To identify factors modifying the effects of vitamin D supplementation, we did prespecified subgroup analyses for the outcome of moderate or severe exacerbation rate by extending the one-step meta-analysis framework to include treatment-covariate interaction terms. Subgroups were defined according to baseline vitamin $\mathrm{D}$ status (serum $25(\mathrm{OH}) \mathrm{D}<25 \mathrm{nmol} / \mathrm{L}$ vs $\geq 25 \mathrm{nmol} / \mathrm{L}$ ), GOLD spirometric grade ${ }^{2}$ (1/2 vs $3 / 4$ ), concomitant use of inhaled corticosteroids (yes vs no), body mass index $\left(<25 \mathrm{~kg} / \mathrm{m}^{2}\right.$ vs $\left.\geq 25 \mathrm{~kg} / \mathrm{m}^{2}\right)$, frequency of vitamin D dosing (intermittent bolus vs daily), genotypes for rs731236, rs11568820, rs7041 and rs4588 (major homozygous vs heterozygous vs minor homozygous) and genotype for Gc (Gc1/1 vs Gc2/1 vs Gc2/2, derived from genotypes for rs7041 and rs4588 as previously reported). ${ }^{19}$ All SNPs were analysed using the per-allele method (ie, under an additive model). The $25 \mathrm{nmol} / \mathrm{L}$ cut-off for baseline $25(\mathrm{OH}) \mathrm{D}$ concentration in the subgroup analyses was selected, because it is the threshold for vitamin $\mathrm{D}$ deficiency defined by the UK Department of Health ${ }^{20}$ and because, below this level, vitamin $\mathrm{D}$ supplementation protects most strongly against acute respiratory infections, ${ }^{6}$ which commonly precipitate COPD exacerbation. We also did an exploratory analysis investigating effects in subgroups defined using the $50 \mathrm{nmol} / \mathrm{L}$ and $75 \mathrm{nmol} / \mathrm{L}$ 
cut-offs for baseline circulating 25(OH)D concentration because observational studies have reported that less profound states of vitamin D deficiency associate independently with increased risk of acute respiratory infection in people with COPD. ${ }^{21}$ Statistical significance was inferred for subgroup effects in which the $\mathrm{p}$ value for the treatment-covariate interaction terms ( $p$ for interaction) was less than 0.05 . Race or ethnicity was not investigated as a potential effect modifier, as there was insufficient ethnic/ racial diversity to power such a subgroup analysis $(97.0 \%$ of participants were of white European origin). Influenza vaccination status was not investigated as a potential effect modifier, as data were unavailable for $48.8 \%$ of participants. We did a responder analysis in participants assigned to the intervention arm of included studies, comparing incidence of moderate or severe COPD exacerbations in participants who attained an end-study serum $25(\mathrm{OH}) \mathrm{D}$ concentration of $75 \mathrm{nmol} / \mathrm{L}$ or higher versus participants who did not.

\section{Quality assessment across studies}

We used the five GRADE considerations (study limitations, consistency of effect, imprecision, indirectness and publication bias) ${ }^{22}$ to assess the quality of the body of evidence contributing to the principal analyses of rate of moderate exacerbations, the proportion of participants with at least one exacerbation requiring emergency department attendance or hospital admission, or both, and the proportion of participants with at least one serious adverse event.

\section{Role of the funding source}

The National Institute of Health Research had no role in study design, data collection, data analysis, or data interpretation, or writing of the report. The corresponding author had full access to all the data in the study and had final responsibility for the decision to submit for publication.

\section{RESULTS}

\section{Study selection and IPD obtained}

Our search identified 187 unique studies that we assessed for eligibility, of which four studies ${ }^{13-16}$ with a total of 560 randomised participants fulfilled eligibility criteria (figure 1). We sought IPD for all four studies and obtained outcome data for $469 / 472$ participants in three studies ${ }^{13-15}$; data were not obtained for one study (88 participants) ${ }^{16}$ because the corresponding author advised that the study database had been lost.

\section{Study and participant characteristics}

The three analysed RCTs were done in different countries (Belgium, the UK and the Netherlands); randomised participants were aged $40-86$ years, $315 / 472(66.7 \%)$ were men and $458 / 472$ (97.0\%) were of white European ethnic origin (table 1).

Baseline serum 25(OH)D concentrations ranged from undetectable to $160 \mathrm{nmol} / \mathrm{L}$ (to convert to $\mathrm{ng} / \mathrm{ml}$, divide by 2.496 ). One of the studies ${ }^{13}$ exclusively enrolled patients who had previously been hospitalised for COPD exacerbation; this study contributed 182/472 (38.6\%) of participants to the meta-analysis, driving the relatively high rate of moderate/severe exacerbations that we report overall. Baseline characteristics of participants randomised to intervention and control arms of included trials were similar (online supplementary appendix table 1 ). All studies administered oral vitamin $\mathrm{D}_{3}$ to participants in the intervention arm; this was given as 12 monthly bolus doses over 1 year in one study (100 000 IU per bolus; total 1200000 IU over 1 year) ${ }^{13}$; as six bolus doses every 2 months over 1 year in another study (120
000 IU per bolus; total 720000 IU over 1 year $)^{14}$; and as a daily dose for 6 months in another study (1200 IU per day; total 220 000 IU over 6 months). ${ }^{15}$ IPD integrity was confirmed by replication of primary analyses in published papers where applicable. The process of checking IPD revealed one discrepancy with a primary report. In the trial by Lehouck and colleagues, ${ }^{13}$ a total of 468 COPD moderate or severe exacerbations were originally reported to have arisen during follow-up. Data extraction for the current meta-analysis identified an additional 33 moderate or severe exacerbations that were not included in the original trial report, bringing the total number of exacerbations occurring in this study to 501 . These additional events represent instances in which exacerbations originally classified as being a single long event were found to comprise two separate exacerbations, as systemic corticosteroids and/or antibiotics were discontinued and restarted.

\section{Risk of bias within studies}

Details of the risk of bias assessment are provided in online supplementary appendix table 2 . All RCTs contributing data to this meta-analysis were assessed as being at low risk of bias for all aspects considered. The trial by Zendedel et al ${ }^{16}$ which did not contribute data to this meta-analysis, was assessed as being at unclear risk of bias for sequence generation, allocation concealment, blinding of participants and personnel, blinding of outcome assessment, incomplete outcome data and selective reporting, and as being at high risk of other bias (validity of primary outcome depended on detailed recall of day-to-day symptoms without use of a diary at 2-month intervals).

\section{Overall results: rate of moderate or severe COPD exacerbations}

Overall, in the one-step IPD meta-analysis, vitamin D supplementation did not influence the rate of moderate or severe COPD exacerbations (adjusted incidence rate ratio (aIRR) 0.94, $95 \%$ CI 0.78 to $1.13 ; \mathrm{p}=0.52 ; 469$ participants in three studies; table 2).

This evidence was assessed as being of high quality (online supplementary appendix table 3). The two-step IPD meta-analysis revealed a similar estimate of the overall effect (aIRR 0.94, $95 \%$ CI 0.79 to $1.12 ; \mathrm{p}=0.51 ; \mathrm{P}$ for heterogeneity $=0.72 ; 469$ participants in three studies; figure 2).

\section{Results of subgroup analyses: rate of moderate or severe COPD exacerbations}

We did prespecified subgroup analyses to investigate whether the effects of vitamin D supplementation on the rate of moderate or severe COPD exacerbations differed according to vitamin D status at baseline, GOLD spirometric grade at baseline, concomitant use of inhaled corticosteroids at baseline, body mass index at baseline, frequency of vitamin D dosing and genotype for SNPs in VDR (rs731236 and rs11568820) and DBP (rs7041, rs4588 and Gc; table 2). Vitamin D supplementation significantly reduced the rate of moderate or severe COPD exacerbations in individuals with baseline $25(\mathrm{OH}) \mathrm{D}$ levels $<25 \mathrm{nmol} / \mathrm{L}$ (aIRR 0.55 , $95 \%$ CI 0.36 to $0.84 ; 87$ participants in three studies; within sub-group $\mathrm{P}=0.006$; moderate quality evidence) but not in those with baseline $25(\mathrm{OH}) \mathrm{D}$ levels $\geq 25 \mathrm{nmol} / \mathrm{L}$ (aIRR $1.04,95 \% \mathrm{CI}$ 0.85 to $1.27 ; 382$ participants in three studies; within sub-group: $\mathrm{p}=0.71 ; \mathrm{p}$ for interaction $=0.015$; high-quality evidence; online supplementary appendix table 3 ). An exploratory subgroup analysis in which baseline $25(\mathrm{OH}) \mathrm{D}$ concentrations were categorised in narrower strata revealed no statistically significant effect of 


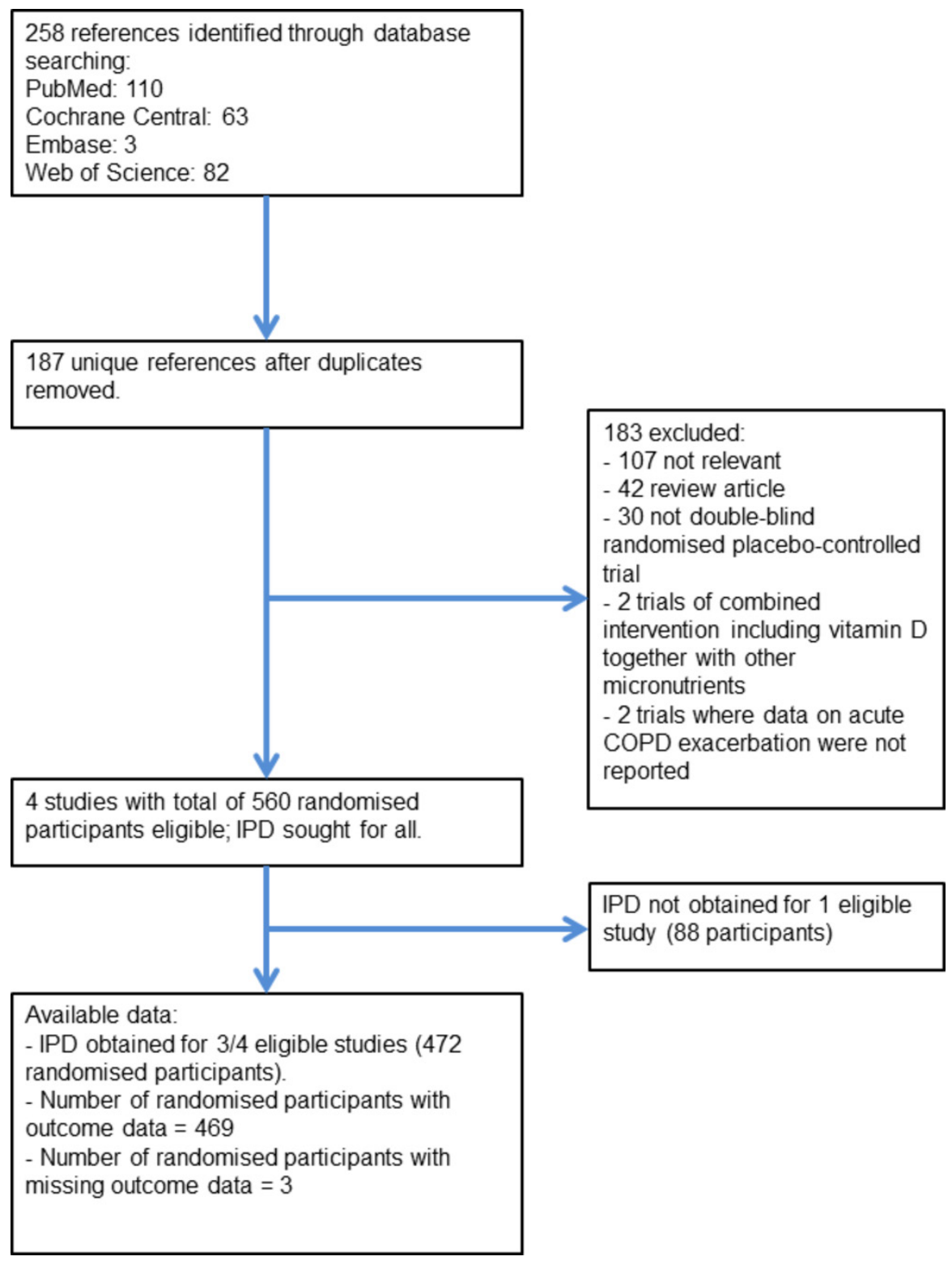

Figure 1 PRISMA diagram of study selection. IPD, individual participant data; PRISMA, Preferred Reporting Items for Systematic Reviews and MetaAnalyses.

vitamin D supplementation on COPD exacerbation rate within subgroups having baseline 25(OH)D 25.0-49.9 nmol/L (aIRR $1.00,95 \%$ CI 0.77 to $1.31,214$ participants in three studies, within sub-group: $\mathrm{p}=0.97$ ), $50.0-74.9 \mathrm{nmol} / \mathrm{L}$ (aIRR 1.06, 95\% CI 0.70 to $1.61 ; 105$ participants in three studies, within sub-group $\mathrm{P}=0.78$ ) or $\geq 75 \mathrm{nmol} / \mathrm{L}$ (aIRR $1.17,95 \% \mathrm{CI} 0.73$ to $1.87,63$ participants in two studies, within sub-group $\mathrm{P}=0.52$; $\mathrm{P}$ for interaction $=0.06$; figure 3 ).

\section{Secondary outcomes: efficacy}

Vitamin D supplementation did not have any statistically significant effect on the proportion of participants experiencing at least one moderate or severe COPD exacerbation; the time to first moderate or severe COPD exacerbation; the proportion of participants experiencing one or more severe COPD exacerbation; or the mean end-study values of $\%$ predicted $\mathrm{FEV}_{1}, \%$ predicted FVC or body mass index (table 3 ).

\section{Secondary Outcomes: safety}

Vitamin D supplementation did not have any statistically significant effect on the proportion of participants of experiencing at least one serious adverse event of any cause (high-quality evidence); the proportion of participants dying of any cause; the proportion of participants dying of COPD exacerbation or respiratory failure; or the proportion of participants experiencing at least one episode of hypercalcaemia. No incident cases of renal stones were reported (table 3 ).

\section{Risk of bias across studies}

Too few studies were available to justify construction of a funnel plot to assess for publication bias.

\section{Additional analyses}

Results of responder analyses are presented in online supplementary appendix table 4. Among participants randomised to the intervention arm of included studies, no difference in COPD 


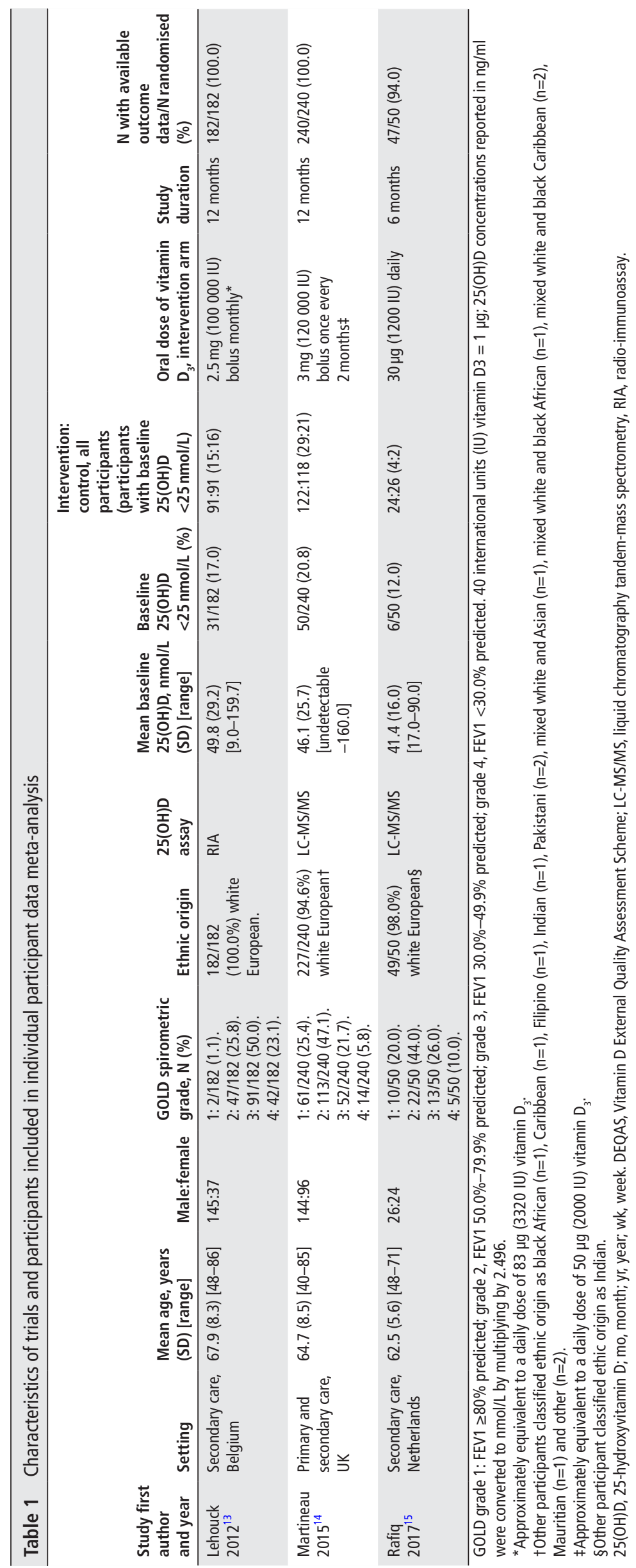


Table 2 One-step individual participant data meta-analysis, rate of moderate or severe COPD exacerbations: overall and by subgroup

\begin{tabular}{|c|c|c|c|c|c|c|}
\hline & $\begin{array}{l}\mathrm{N} \text { individuals } \\
\text { (trials) }\end{array}$ & $\begin{array}{l}\text { Event rate per participant- } \\
\text { year, control group }\end{array}$ & $\begin{array}{l}\text { Event rate per participant-year, } \\
\text { intervention group }\end{array}$ & $\begin{array}{l}\text { Adjusted incidence rate } \\
\text { ratio }(95 \% \mathrm{Cl})^{*}\end{array}$ & $P$ value & $\begin{array}{l}P \text { value for } \\
\text { interaction }\end{array}$ \\
\hline Overall & $469(3)$ & $398 / 201.87(1.97)$ & $381 / 205.66(1.85)$ & 0.94 (0.78 to 1.13$)$ & 0.52 & \\
\hline \multicolumn{7}{|c|}{ Baseline 25(OH)D (nmol/L) } \\
\hline$<25$ & $87(3)$ & $77 / 36.60(2.10)$ & $51 / 41.52(1.23)$ & 0.55 (0.36 to 0.84$)$ & 0.006 & \multirow[t]{2}{*}{0.015} \\
\hline$\geq 25$ & $382(3)$ & $321 / 165.27(1.94)$ & $330 / 164.13(2.01)$ & 1.04 (0.85 to 1.27$)$ & 0.71 & \\
\hline \multicolumn{7}{|c|}{ GOLD spirometric grade } \\
\hline 1 or 2 & $252(3)$ & $139 / 109.17(1.27)$ & $137 / 111.11(1.23)$ & 1.01 (0.75 to 1.35$)$ & 0.94 & \multirow[t]{2}{*}{0.59} \\
\hline 3 or 4 & $217(3)$ & $259 / 92.70(2.79)$ & $244 / 94.55(2.58)$ & $0.91(0.72$ to 1.16$)$ & 0.44 & \\
\hline \multicolumn{7}{|c|}{ Concomitant inhaled corticosteroid at baseline } \\
\hline No & $126(3)$ & $63 / 49.94(1.26)$ & $58 / 56.17(1.03)$ & $0.93(0.56$ to 1.55$)$ & 0.78 & \multirow[t]{2}{*}{0.75} \\
\hline Yes & $343(3)$ & $335 / 151.93(2.20)$ & $323 / 149.49(2.16)$ & 0.96 (0.79 to 1.16$)$ & 0.66 & \\
\hline \multicolumn{7}{|c|}{ Body mass index $\left(\mathrm{kg} / \mathrm{m}^{2}\right)$} \\
\hline$<25$ & $213(3)$ & $210 / 103.67(2.03)$ & $173 / 83.89(2.06)$ & $1.03(0.78$ to 1.36$)$ & 0.84 & \multirow[t]{2}{*}{0.53} \\
\hline$\geq 25$ & $256(3)$ & $188 / 98.20(1.91)$ & $208 / 121.77(1.71)$ & $0.89(0.70$ to 1.15$)$ & 0.38 & \\
\hline \multicolumn{7}{|c|}{ Frequency of vitamin $D$ dosing } \\
\hline Intermittent bolus & $422(2)$ & $374 / 189.75(1.97)$ & $364 / 193.67(1.88)$ & $0.96(0.79$ to 1.15$)$ & 0.64 & \multirow[t]{2}{*}{0.43} \\
\hline Daily & $47(1)$ & $24 / 12.12(1.98)$ & $17 / 11.98(1.42)$ & 0.74 (0.38 to 1.41$)$ & 0.35 & \\
\hline \multicolumn{7}{|c|}{ rs731236 (Taql, VDR) genotype } \\
\hline $\mathrm{TT}$ & $142(2)$ & $105 / 57.09(1.84)$ & $119 / 71.18(1.67)$ & 0.95 (0.70 to 1.31$)$ & 0.77 & \multirow[t]{3}{*}{0.52} \\
\hline СT & $198(2)$ & $184 / 89.80(2.05)$ & $163 / 89.20(1.83)$ & 0.90 (0.67 to 1.19$)$ & 0.45 & \\
\hline CC & $67(2)$ & $65 / 37.75(1.72)$ & $65 / 25.95(2.51)$ & $1.28(0.81$ to 2.02$)$ & 0.28 & \\
\hline \multicolumn{7}{|c|}{ rs11568820 (cdx2, VDR) genotype } \\
\hline GG & $234(2)$ & $190 / 105.35(1.80)$ & $196 / 104.79(1.87)$ & 0.99 (0.77 to 1.28$)$ & 0.95 & \multirow[t]{3}{*}{0.72} \\
\hline GA & $149(2)$ & $151 / 67.80(2.23)$ & $130 / 71.22(1.83)$ & 0.92 (0.68 to 1.25$)$ & 0.59 & \\
\hline AA & $20(2)$ & $13 / 9.20(1.41)$ & $12 / 7.86(1.53)$ & $1.41(0.55$ to 3.66$)$ & 0.48 & \\
\hline \multicolumn{7}{|c|}{ rs7041 (Haelll, DBP) genotype } \\
\hline GG & $106(2)$ & $108 / 48.67(2.22)$ & $106 / 47.72(2.22)$ & 1.08 (0.78 to 1.49$)$ & 0.66 & \multirow[t]{3}{*}{0.15} \\
\hline GT & $207(2)$ & $157 / 88.47(1.77)$ & $182 / 99.11(1.84)$ & $1.05(0.78$ to 1.40$)$ & 0.77 & \\
\hline $\mathrm{TT}$ & $92(2)$ & $89 / 46.18(1.93)$ & $63 / 38.92(1.62)$ & 0.75 (0.51 to 1.09$)$ & 0.14 & \\
\hline \multicolumn{7}{|c|}{ rs4588 (Styl, DBP) genotype } \\
\hline CC & $191(2)$ & $175 / 91.53(1.91)$ & $168 / 83.16(2.02)$ & 1.05 (0.80 to 1.39$)$ & 0.72 & \multirow[t]{3}{*}{0.34} \\
\hline $\mathrm{CA}$ & $184(2)$ & $151 / 77.08(1.96)$ & $158 / 90.00(1.76)$ & $0.93(0.70$ to 1.24$)$ & 0.61 & \\
\hline AA & $35(2)$ & $31 / 16.95(1.83)$ & $25 / 14.45(1.73)$ & 0.84 (0.41 to 1.73$)$ & 0.64 & \\
\hline \multicolumn{7}{|l|}{$\mathrm{Gc}(D B P)$ genotype } \\
\hline $\mathrm{Gc} 1 / 1$ & $187(2)$ & $172 / 89.45(1.92)$ & $164 / 81.14(2.02)$ & 1.07 (0.80 to 1.42$)$ & 0.65 & \multirow[t]{3}{*}{0.24} \\
\hline $\mathrm{Gc} 2 / 1$ & $183(2)$ & $151 / 77.08(1.96)$ & $158 / 89.16(1.77)$ & $0.93(0.70$ to 1.24$)$ & 0.63 & \\
\hline $\mathrm{Gc} 2 / 2$ & $32(2)$ & $31 / 16.78(1.85)$ & $18 / 12.62(1.43)$ & 0.68 (0.29 to 1.58$)$ & 0.37 & \\
\hline
\end{tabular}

GOLD spirometric grade 1 or $2: \mathrm{FEV}_{1} \geq 50 \%$ predicted; grade 3 or $4: \mathrm{FEV}_{1}<50 \%$ predicted. ${ }^{*}$, adjusted for age, sex, COPD severity and trial.

25(OH)D, 25-hydroxyvitamin D; DBP, vitamin D binding protein; Gc, group-specific component; GOLD, Global Initiative for Chronic Obstructive Lung Disease; VDR, vitamin D receptor.

exacerbation incidence was observed between participants who attained end-study serum $25(\mathrm{OH}) \mathrm{D}$ concentrations $\geq 75 \mathrm{nmol} / \mathrm{L}$ $(\mathrm{n}=138)$ versus those who did not $(\mathrm{n}=66)$.

\section{DISCUSSION}

We report results of the first meta-analysis of RCTs of vitamin $\mathrm{D}$ to reduce the risk of COPD exacerbations. In the study population as a whole, vitamin $\mathrm{D}$ did not influence the rate of moderate or severe COPD exacerbations as compared with placebo (1.85 vs 1.97 events per person per year; $\mathrm{p}=0.52$ ). Subgroup analysis revealed that vitamin D supplementation, compared with placebo, reduced the rate of moderate or severe COPD exacerbations in participants with a baseline $25(\mathrm{OH}) \mathrm{D}$ of less than $25 \mathrm{nmol} / \mathrm{L}$ (1.23 vs 2.10 events per person per year; $p=0.006)$, but not in those with a baseline serum $25(\mathrm{OH}) \mathrm{D}$ concentration of $25 \mathrm{nmol} / \mathrm{L}$ or higher $(2.01$ vs 1.94 events per person per year, $p=0.71 ; p$ for interaction $=0.015)$. Vitamin D supplementation was safe at the doses administered: serious adverse events and occasional episodes of hypercalcaemia were evenly distributed between participants randomised to vitamin $\mathrm{D}$ versus placebo, and no instances of renal stones were seen. 


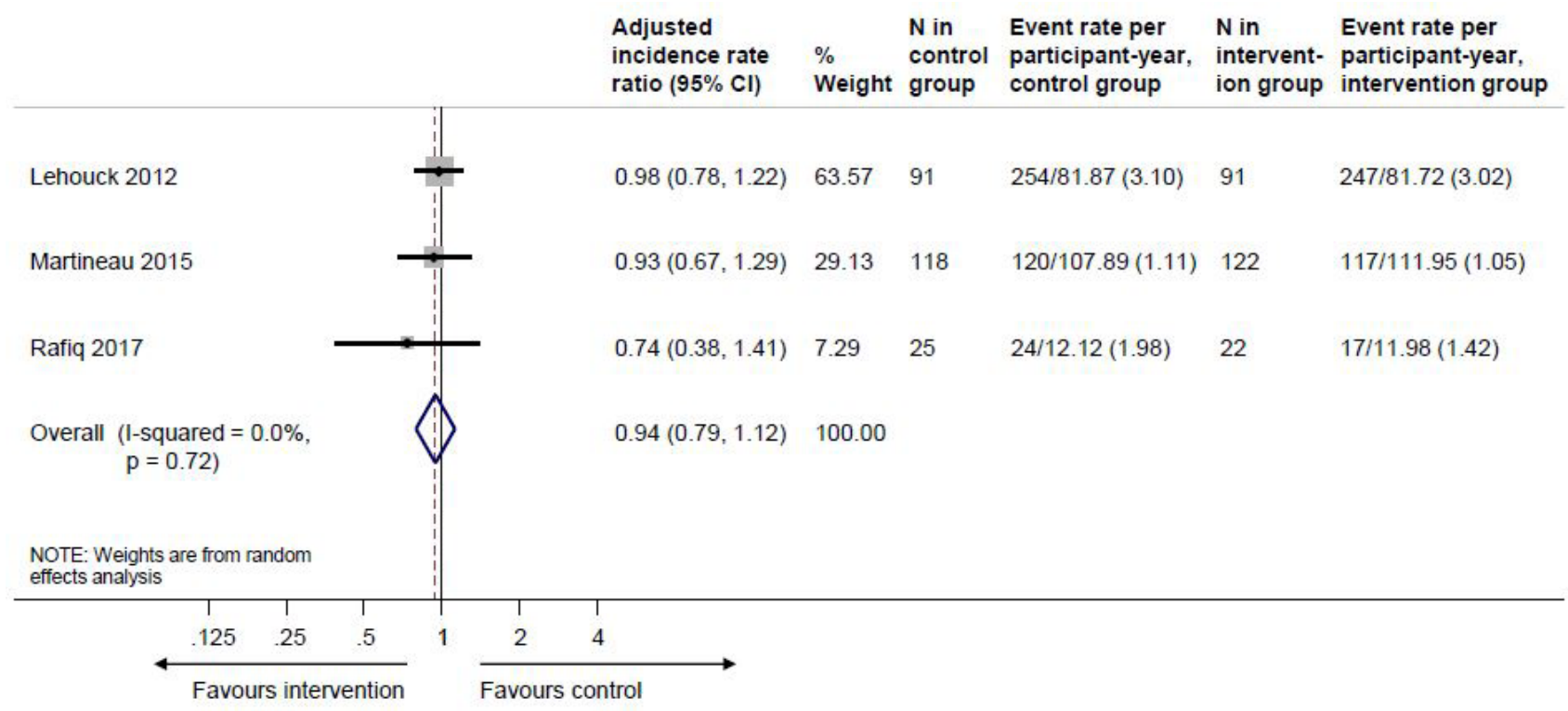

Figure 2 Two-step individual participant data meta-analysis, rate of moderate or severe COPD exacerbations. Incidence rate ratios are adjusted for age, sex and GOLD spirometric grade. GOLD, Global Initiative for Chronic Obstructive Lung Disease.

Our finding that protective effects of vitamin D supplementation against COPD exacerbation are restricted to those with the lowest baseline $25(\mathrm{OH}) \mathrm{D}$ concentrations echoes results of our recent IPD meta-analysis of RCTs of vitamin D for the prevention of asthma exacerbation, in which we found a statistically significant protective effect of supplementation in those with $25(\mathrm{OH}) \mathrm{D}<25 \mathrm{nmol} / \mathrm{L}$, but not in those with higher baseline vitamin D status. ${ }^{7}$ We have also previously reported that protective effects of vitamin D supplementation against acute respiratory infection are strongest in those with baseline $25(\mathrm{OH}) \mathrm{D}<25 \mathrm{nmol} / \mathrm{L}^{6}$ Since acute respiratory infections commonly precipitate exacerbations of both asthma and COPD, and it may be that protective effects of vitamin D against these outcomes are mediated by a common mechanism,

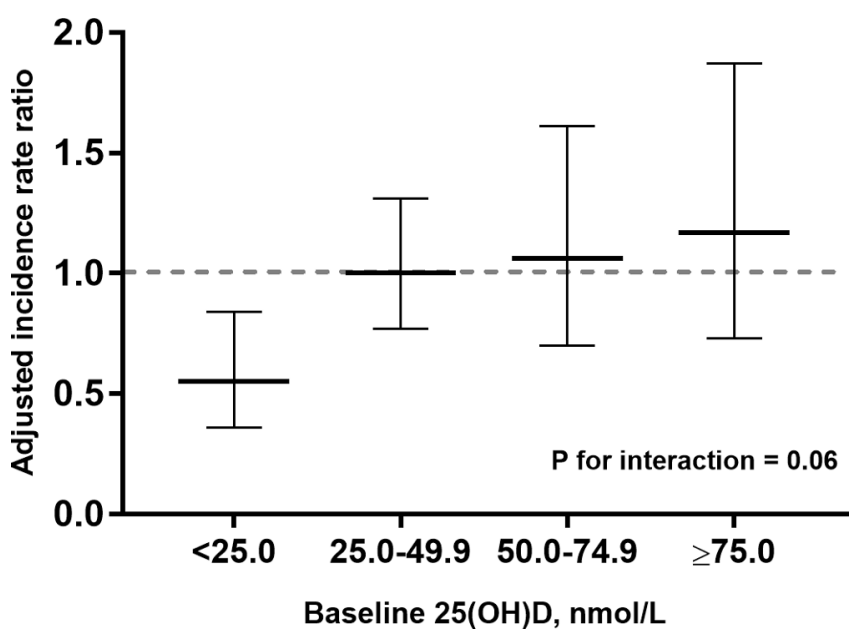

Figure 3 Effects of vitamin D supplementation on rate of moderate or severe COPD exacerbations by baseline circulating 25-hydroxyvitamin D concentration, categorised by $25 \mathrm{nmol} / \mathrm{L}$ strata: results of one-step individual participant data meta-analysis. Incidence rate ratio adjusted for age, sex and GOLD spirometric grade. Bar and whiskers represent mean and 95\% Cl respectively. 25(OH)D, 25- hydroxyvitamin D; GOLD, Global Initiative for Chronic Obstructive Lung Disease. namely induction of antiviral and antimicrobial responses. The protective effects demonstrated in this meta-analysis of intervention studies are consistent with results of some observational studies ${ }^{9}$ but at variance with others ${ }^{10}$ : failure to demonstrate an association between vitamin $\mathrm{D}$ deficiency and increased exacerbation risk in some observational studies may relate to the low prevalence of participants with baseline $25(\mathrm{OH}) \mathrm{D}$ levels $<25 \mathrm{nmol} / \mathrm{L}^{23}$

One puzzling finding is that exacerbation rates among control arm participants were similar for those with lower versus higher baseline $25(\mathrm{OH}) \mathrm{D}$ levels (2.10 vs 1.94 events per participant-year, respectively); in the intervention arm, the exacerbation rate was reduced in those with lower versus higher baseline $25(\mathrm{OH}) \mathrm{D}$ levels $(1.23 \mathrm{vs}$ 2.01 , respectively). This phenomenon may reflect heterogeneity in factors driving exacerbations-a concept that is well recognised. ${ }^{24}$ We hypothesise that exacerbations in the vitamin D-deficient group may be largely driven by vitamin D deficiency; hence, vitamin D replacement reduces exacerbation risk in this group. By contrast, in groups with higher baseline vitamin D levels, factors other than vitamin D deficiency (eg, inherently increased viral susceptibility, or exposure to an as-yet uncharacterised environmental factor) may be the primary drivers of exacerbation risk.

Our study has several strengths: we included data from three out of four eligible studies (84\% of participants worldwide), all of which were assessed as being at low risk of bias. Access to IPD, with detailed characterisation of potential environmental and genetic effect modifiers, allowed for comprehensive prespecified subgroup analyses to determine whether effects of vitamin D supplementation varied between individuals. Included studies recruited patients with mild, moderate and severe disease from primary and secondary care in three different countries, enhancing generalisability of our results.

Our study also has some limitations. It incorporates data from a modest number of trials-too few to justify construction of a funnel plot to formally assess for publication bias. Our findings should therefore be interpreted with caution. We were unable to obtain IPD for one relatively small $(n=88)$ eligible study ${ }^{16}$; data from this study could not be included in an aggregate data meta-analysis, since summary statistics 


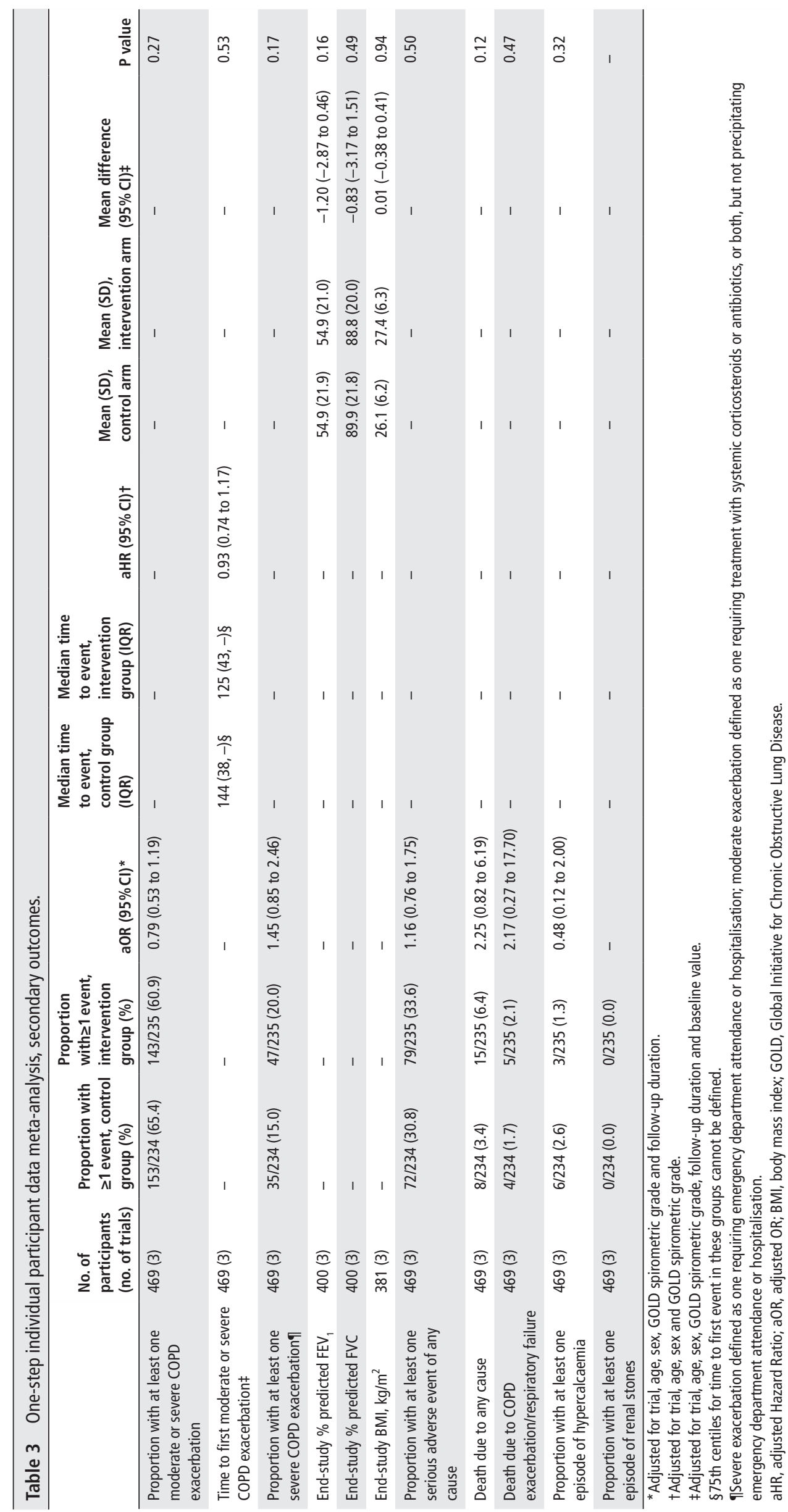


relating to the rate of exacerbations, the proportion of participants experiencing at least one exacerbation or the time to first exacerbation were not reported. It is questionable, however, whether this study would have contributed useful data to our meta-analysis, since use of antibiotics/systemic corticosteroids was not reported, vitamin D status of participants was not measured and the validity of assessments of exacerbation was assessed as being at high risk of bias. Since the study was small and it reported a protective effect of vitamin D supplementation against exacerbations, the effect of its omission, if any, may have been to slightly bias results of this meta-analysis towards the null. We are aware of at least one ongoing RCT of vitamin D supplementation for the prevention of COPD exacerbations ${ }^{25}(n=240$, scheduled to complete follow-up in 2019). Inclusion of IPD from this study and other eligible studies in a future meta-analysis has potential to increase power for subgroup analyses and generalisability of results.

Our study showed no overall effect of vitamin D supplementation on the rate of moderate or severe COPD exacerbations. However, subgroup analysis revealed clinically and statistically significant protective effects of vitamin D supplementation among patients with baseline circulating $25(\mathrm{OH}) \mathrm{D}$ concentrations of less than $25 \mathrm{nmol} / \mathrm{L}$. Given the high prevalence of profound vitamin D deficiency in people with COPD, ${ }^{26}$ and the large reduction in exacerbation rates seen with vitamin D supplementation in this group, our findings support a strategy of routinely testing vitamin D status in patients with COPD who experience exacerbations and offering supplementation to those with circulating $25(\mathrm{OH}) \mathrm{D}$ concentrations of less than $25 \mathrm{nmol} / \mathrm{L}$.

\section{Author affiliations \\ ${ }^{1}$ Centre for Primary Care and Public Health, Barts and The London School of Medicine and Dentistry, Queen Mary University of London, London, UK ${ }^{2}$ Department of Respiratory Medicine, Katholieke Universiteit, Leuven, Belgium ${ }^{3}$ Department of Internal Medicine and Endocrinology, VU University Medical Center, Amsterdam, The Netherlands \\ ${ }^{4}$ Department of Emergency Medicine, Massachusetts General Hospital, Harvard Medical School, Boston, Massachusetts, UK \\ ${ }^{5}$ Asthma UK Centre for Applied Research, Blizard Institute, Queen Mary University of London, London, UK}

\begin{abstract}
Acknowledgements We would like thank all the people who participated in primary RCTs; the teams who conducted them; and our patient and public involvement representatives Mrs Charanjit Patel and Ms Jane Gallagher for comments on study design and drafts of this manuscript.
\end{abstract}

Contributors ARM led the funding application, with input from RLH, CAC and CJG who were coapplicants. ARM, DAJ and WJ assessed eligibility of studies for inclusion. ARM, DAJ, CM, RR, RTdJ and WJ were all directly involved in the acquisition of data for the work. RLH designed statistical analyses in consultation with authors contributing individual patient data. Statistical analyses were done by DAJ, LG and RLH. ARM wrote the first draft of the report. All authors revised it critically for important intellectual content, gave final approval of the version to be published and agreed to be accountable for all aspects of the work in ensuring that questions related to the accuracy or integrity of any part of the work were appropriately investigated and resolved.

Funding This research was supported by a grant from National Institute for Health Research (NIHR) under its Health Technology Assessment (HTA) Programme (Reference Number 13/03/25 to ARM).

Disclaimer The views expressed are those of the authors and not necessarily those of the National Health Service, the NIHR or the Department of Health.

Competing interests None declared.

Patient consent for publication Not required.
Provenance and peer review Not commissioned; externally peer reviewed.

Data sharing statement A partial dataset, incorporating patient level data from RCTs for which the relevant permission for data sharing have been obtained, is available from the corresponding author at a.martineau@qmul.ac.uk.

\section{REFERENCES}

1 GBD 2015 Chronic Respiratory Disease Collaborators. Global, regional, and national deaths, prevalence, disability-adjusted life years, and years lived with disability for chronic obstructive pulmonary disease and asthma, 1990-2015: a systematic analysis for the Global Burden of Disease Study 2015. Lancet Respir Med 2017;5:691-706.

2 Global Initiative for Chronic Obstructive Lung Disease (GOLD). Global Strategy for the Diagnosis, Management and Prevention of COPD, 2017. http://goldcopd.org.

3 Wedzicha JA, Seemungal TA. COPD exacerbations: defining their cause and prevention. Lancet 2007;370:786-96.

4 Greiller CL, Martineau AR. Modulation of the immune response to respiratory viruses by vitamin D. Nutrients 2015;7:4240-70.

5 Greiller CL, Suri R, Jolliffe DA, et al. Vitamin D attenuates rhinovirus-induced expression of intercellular adhesion molecule-1 (ICAM-1) and platelet-activating factor receptor (PAFR) in respiratory epithelial cells. J Steroid Biochem Mol Biol 2018.

6 Martineau AR, Jolliffe DA, Hooper RL, et al. Vitamin D supplementation to prevent acute respiratory tract infections: systematic review and meta-analysis of individual participant data. BMJ 2017;356:i6583.

7 Jolliffe DA, Greenberg L, Hooper RL, et al. Vitamin D supplementation to prevent asthma exacerbations: a systematic review and meta-analysis of individual participant data. Lancet Respir Med 2017;5:881-90.

8 Janssens W, Decramer M, Mathieu C, et al. Vitamin D and chronic obstructive pulmonary disease: hype or reality? Lancet Respir Med 2013;1:804-12.

9 Malinovschi A, Masoero M, Bellocchia M, et al. Severe vitamin D deficiency is associated with frequent exacerbations and hospitalization in COPD patients. Respir Res 2014;15:131

10 Kunisaki KM, Niewoehner DE, Connett JE. COPD Clinical Research Network. Vitamin D levels and risk of acute exacerbations of chronic obstructive pulmonary disease: a prospective cohort study. Am J Respir Crit Care Med 2012;185:286-90.

11 Quint JK, Donaldson GC, Wassef N, et al. 25-hydroxyvitamin D deficiency, exacerbation frequency and human rhinovirus exacerbations in chronic obstructive pulmonary disease. BMC Pulm Med 2012;12:28.

12 Puhan MA, Siebeling L, Frei A, et al. No association of 25-hydroxyvitamin D with exacerbations in primary care patients with COPD. Chest 2014;145:37-43.

13 Lehouck A, Mathieu C, Carremans C, et al. High doses of vitamin D to reduce exacerbations in chronic obstructive pulmonary disease: a randomized trial. Ann Intern Med 2012;156:105-14

14 Martineau AR, James WY, Hooper RL, et al. Vitamin D3 supplementation in patients with chronic obstructive pulmonary disease (ViDiCO): a multicentre, double-blind, randomised controlled trial. Lancet Respir Med 2015;3:120-30.

15 Rafiq R, Prins HJ, Boersma WG, et al. Effects of daily vitamin D supplementation on respiratory muscle strength and physical performance in vitamin D-deficient COPD patients: a pilot trial. Int I Chron Obstruct Pulmon Dis 2017;12:2583-92.

16 Zendedel A, Gholami M, Anbari K, et al. Effects of vitamin d intake on fev1 and copd exacerbation: A randomized clinical trial study. Glob J Health Sci 2015;7:243-8.

17 Stewart LA, Clarke M, Rovers M, et al. Preferred reporting items for systematic review and meta-analyses of individual participant data: the PRISMA-IPD statement. JAMA 2015;313:1657-65.

18 Riley RD, Lambert PC, Abo-Zaid G. Meta-analysis of individual participant data: rationale, conduct, and reporting. BMJ 2010;340:c221.

19 Martineau AR, Leandro AC, Anderson ST, et al. Association between Gc genotype and susceptibility to TB is dependent on vitamin D status. Eur Respir J 2010;35:1106-12.

20 Department of Health. Department of Health Report on Health and Social Subjects, No. 49. Nutrition and bone health with particular reference to calcium and vitamin D. London 1998.

21 Ginde AA, Mansbach JM, Camargo CA. Association between serum 25-hydroxyvitamin D level and upper respiratory tract infection in the Third National Health and Nutrition Examination Survey. Arch Intern Med 2009;169:384-90.

22 Guyatt GH, Oxman AD, Vist GE, et al. GRADE: an emerging consensus on rating quality of evidence and strength of recommendations. BMJ 2008;336:924-6.

23 Shaheen SO, Martineau AR. Vitamin D and chronic obstructive pulmonary disease: justified optimism or false hope? Am J Respir Crit Care Med 2012;185:239-41.

24 Sapey E, Stockley RA. COPD exacerbations. 2: aetiology. Thorax 2006;61:250-8.

25 Rafiq R, Aleva FE, Schrumpf JA, et al. Prevention of exacerbations in patients with COPD and vitamin D deficiency through vitamin D supplementation (PRECOVID): a study protocol. BMC Pulm Med 2015;15:106.

26 Jolliffe DA, James WY, Hooper RL, et al. Prevalence, determinants and clinical correlates of vitamin $D$ deficiency in patients with Chronic Obstructive Pulmonary Disease in London, UK. J Steroid Biochem Mol Biol 2018;175:138-45. 